PR-24

\title{
SYNTHESIS OF HETEROARYL UREA DERIVATIVES AS ANTIMICROBIAL AGENTS
}

\author{
K. Narendra Babu, ${ }^{1}$ G. Sravya, ${ }^{2}$ N. Hussain Basha, ${ }^{1}$ Grigory. V. Zyryanov, ${ }^{2,3}$ V. Padmavathi ${ }^{1 *}$ \\ ${ }^{1}$ Department of Chemistry, Sri Venkateswara University, Tirupati, Andhra Pradesh,India. \\ ${ }^{2}$ Ural Federal University, Chemical Engineering Institute, Yekaterinburg, \\ 620002, Russian Federation.
}

${ }^{3}$ I. Ya. Postovsky Institute of Organic Synthesis, Ural Division of Russian Academy of Sciences, 22 S. Kovalevskoy St., 620219, Yekaterinburg, Russian Federation.

*Corresponding author, E-mail: vkpuram2001@yahoo.com

\begin{abstract}
Azoles are prominent scaffolds in pharmaceutical arena. In fact, medicinal properties of azole and benzazole containing compounds include anticancer, antimicrobial, and antioxidant. Some of the drugs Inthomycin C, Oxaprozin, Tiazofurin, Dacarbazine, Tipifarnib, Albendazole, Febendazole, Omeprazole possess azole / benzazole moiety. Realizing the importance of azoles and benzazoles, it is planned to conjugate these two ligands as heteroaryl substituted urea derivatives and to study their antimicrobial activity. The results pertaining to these aspects will be discussed.
\end{abstract}

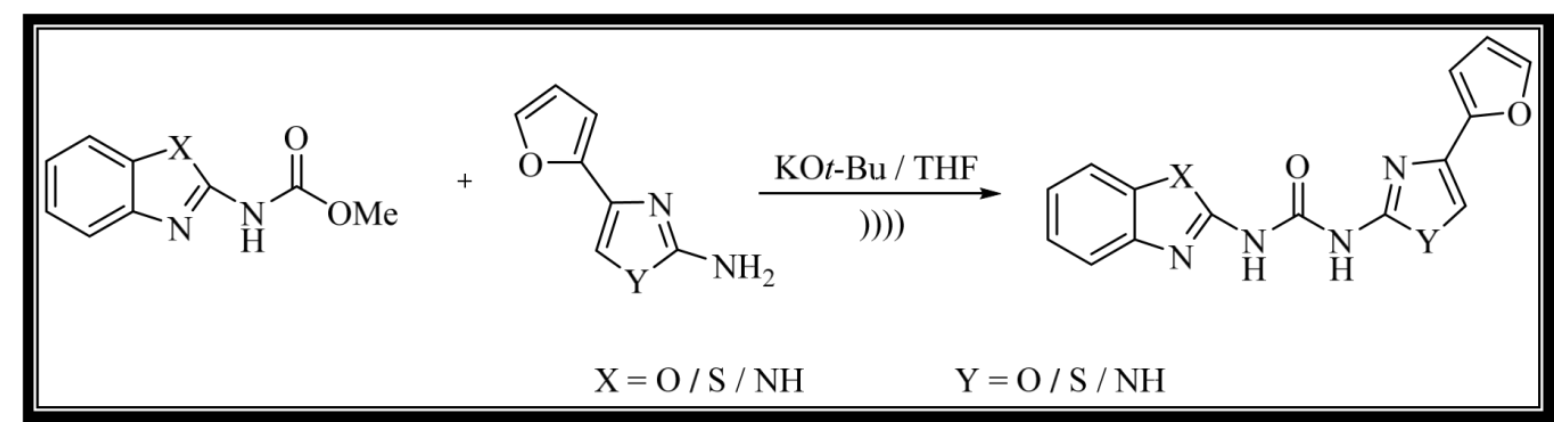

